PROCEEDINGS OF THE

AMERICAN MATHEMATICAL SOCIETY

Volume 127, Number 1, January 1999, Pages 45-50

S 0002-9939(99)04584-0

\title{
LARGE ORBITS IN ACTIONS OF NILPOTENT GROUPS
}

\author{
I. M. ISAACS
}

(Communicated by Ronald M. Solomon)

\begin{abstract}
If a nontrivial nilpotent group $N$ acts faithfully and coprimely on a group $H$, it is shown that some element of $H$ has a small centralizer in $N$ and hence lies in a large orbit. Specifically, there exists $x \in H$ such that $\left|\mathbf{C}_{N}(x)\right| \leq(|N| / p)^{1 / p}$, where $p$ is the smallest prime divisor of $|N|$.
\end{abstract}

\section{INTRODUCTION}

A well known result of D. S. Passman [3] asserts that if a $p$-group $P$ acts faithfully via automorphisms on a solvable $p^{\prime}$-group $H$, then there must always exist a 'large' orbit. Equivalently, there always exists $x \in H$ such that $\mathbf{C}_{P}(x)$ is 'small', and in most cases, $x$ can be chosen so that $\mathbf{C}_{P}(x)=1$ and $x$ lies in a regular $P$-orbit. Specifically, Passman's result is that if $p=2$, then there exists $x \in H$ such that $\left|\mathbf{C}_{P}(x)\right| \leq|P|^{1 / 2}$, and if $p>2$, one can find $x \in H$ with $\left|\mathbf{C}_{P}(x)\right| \leq|P|^{1 / 3}$. In fact, Passman showed that the only situations where a regular orbit can fail to exist are when $p=2$ and $|H|$ is divisible by a Fermat or Mersenne prime, or when $p$ is Mersenne and $|H|$ is even.

At the end of his paper, Passman stated without proof that in all cases, there exists $x \in H$ such that $\left|\mathbf{C}_{P}(x)\right| \leq|P|^{1 / p}$. The main purpose of this note is to provide a proof of this assertion, with a slightly improved bound.

Theorem A. Let $P$ be a nontrivial p-group that acts faithfully on a group $H$, where $|H|$ is not divisible by $p$. Then there exists an element $x \in H$ such that $\left|\mathbf{C}_{P}(x)\right| \leq(|P| / p)^{1 / p}$.

Notice that we are not assuming that $H$ is solvable. No such hypothesis is necessary because of an amazing elementary lemma of B. Hartley and A. Turull that applies whenever $A$ acts by automorphisms on $G$, where $A$ and $G$ are finite groups having coprime orders. The Hartley-Turull lemma (see Lemma 2.6.2 of [2]) asserts that it is always possible to replace $G$ by an abelian group $G_{0}$ such that the actions of $A$ on the elements of $G$ and on the elements of $G_{0}$ are permutation isomorphic. In fact, $G_{0}$ can be chosen to have square-free exponent, although we shall not need this additional information.

It is not really necessary in Theorem A that the acting group should be a $p$ group; the corresponding result holds whenever an arbitrary finite nilpotent group acts coprimely. Theorem A, of course, is included in the following.

Received by the editors May 12, 1997.

1991 Mathematics Subject Classification. Primary 20D15.

This research was partially supported by a grant from the National Security Agency.

(C)1999 American Mathematical Society 
Theorem B. Let $N$ be a nontrivial nilpotent $\pi$-group, where $\pi$ is a set of primes, and assume that $N$ acts faithfully on a $\pi^{\prime}$-group $H$. Then there exists $x \in H$ such that $\left|\mathbf{C}_{N}(x)\right| \leq(|N| / p)^{1 / p}$, where $p$ is the smallest member of $\pi$.

Because of the Hartley-Turull lemma mentioned earlier, it is no loss to assume that $H$ is abelian when proving Theorem $\mathrm{B}$, and we will do so. Also, it turns out that only the nonabelian Sylow subgroups of $N$ really matter, and hence Theorem B can be strengthened, as follows.

Theorem C. Let $N$ be nilpotent and suppose that it acts faithfully on $H$, where $(|N|,|H|)=1$. Let $\pi$ be a set of prime numbers containing all primes for which the Sylow subgroup of $N$ is nonabelian. Then there exists an element $x \in H$ such that $\left|\mathbf{C}_{N}(x)\right|$ is a $\pi$-number, and if $\pi$ is nonempty, $x$ can be chosen so that $\left|\mathbf{C}_{N}(x)\right| \leq$ $(k / p)^{1 / p}$, where $k=|N|_{\pi}$ is the $\pi$-part of $|N|$ and $p$ is the smallest member of $\pi$.

By a ' $\pi$-number' in the statement of Theorem $\mathrm{C}$, we mean, of course, a positive integer all of whose prime divisors lie in $\pi$.

\section{Coprimeness consequences and Theorem C}

We begin with an easy lemma.

(2.1) Lemma. Suppose that $N$ acts faithfully on $H=A \times B$, where $A$ and $B$ are $N$-invariant. Let $M=\mathbf{C}_{N}(A)$, so that $N / M$ acts on $A$, and let $a \in A$ and $b \in B$. Write $u=\left|\mathbf{C}_{N / M}(a)\right|$ and $v=\left|\mathbf{C}_{M}(b)\right|$. Then $\left|\mathbf{C}_{N}(a b)\right|$ divides uv.

Proof. Let $U=\mathbf{C}_{N}(a)$, so that $U \supseteq M$ and $|U / M|=u$. Also, let $V=\mathbf{C}_{N}(b)$, so that $|V \cap M|=v$. Then

$$
|V \cap U: V \cap M|=|(V \cap U):(V \cap U) \cap M|=|(V \cap U) M: M|,
$$

and this divides $|U / M|=u$. We now have

$$
\left|\mathbf{C}_{N}(a b)\right|=|V \cap U|=|V \cap U: V \cap M||V \cap M|=|V \cap U: V \cap M| v,
$$

and this divides $u v$, as required.

Assuming Theorem B for the moment, we now prove Theorem C.

Proof of Theorem C. Recall that $N$ is nilpotent and acts faithfully and coprimely on $H$. Also, $\pi$ contains all primes for which a Sylow subgroup of $N$ is nonabelian, and we write $k=|N|_{\pi}$ to denote the $\pi$-part of $|N|$. Furthermore, by the HartleyTurull lemma, we can assume that $H$ is abelian.

We associate a real number $f(r)$ to an arbitrary $\pi$-number $r$ by setting $f(r)=1$ if $r=1$ and $f(r)=(r / p)^{1 / p}$ if $r>1$, where $p$ is the smallest member of $\pi$. Observe that if $r$ and $s$ are $\pi$-numbers, then $f(r) f(s) \leq f(r s)$. Expressed in terms of the function $f$, our goal is to find an element $x \in H$ such that $\left|\mathbf{C}_{N}(x)\right|$ is a $\pi$-number and $\left|\mathbf{C}_{N}(x)\right| \leq f(k)$.

Work by induction on $|H|$, and suppose first that it is possible to write $H=$ $A \times B$, where $A$ and $B$ are proper subgroups of $H$ that admit the action of $N$. Let $M=\mathbf{C}_{N}(A)$ and note that $M$ acts faithfully on $B$ since $N$ is faithful on $H$. Observe that $\pi$ contains all primes for which a Sylow subgroup of $M$ is nonabelian, and write $s=|M|_{\pi}$. Since $|B|<|H|$, the inductive hypothesis guarantees that there exists an element $b \in B$ such that $v=\left|\mathbf{C}_{M}(b)\right|$ is a $\pi$-number with $v \leq f(s)$. 
Also, $\pi$ contains all primes for which a Sylow subgroup of $N / M$ is nonabelian, and we write $r=|N / M|_{\pi}$, so that $r s=k$. Since $|A|<|H|$, we can apply the inductive hypothesis to the faithful action of $N / M$ on $A$, and we deduce that there exists an element $a \in A$ such that $u=\left|\mathbf{C}_{N / M}(a)\right|$ is a $\pi$-number with $u \leq f(r)$. By Lemma 2.1, we know that $\left|\mathbf{C}_{N}(a b)\right|$ divides $u v$, and hence it is a $\pi$-number not exceeding $u v \leq f(r) f(s) \leq f(r s)=f(k)$, as desired.

We can now assume that there is no proper decomposition $H=A \times B$, where $A$ and $B$ admit $N$. Let $z$ be any nonidentity $\pi^{\prime}$-element of $N$ and note that $z \in \mathbf{Z}(N)$, so that both $\mathbf{C}_{H}(z)$ and $[H, z]$ are $N$-invariant. By Fitting's lemma, we have $H=\mathbf{C}_{H}(z) \times[H, z]$, and hence one of these factors must be trivial. Because the action of $N$ on $H$ is faithful, we cannot have $[H, z]=1$, and thus $\mathbf{C}_{H}(z)=1$ for every nonidentity $\pi^{\prime}$-element $z$ of $N$. In other words, $\mathbf{C}_{N}(x)$ is a $\pi$-group for every nonidentity element $x \in H$.

Let $K$ be the Hall $\pi$-subgroup of $N$ and note that $|K|=k$. Since $K$ acts faithfully on $H$, we can apply Theorem $\mathrm{B}$ to this action to find a nonidentity element $x$ in $H$ such that $\left|\mathbf{C}_{K}(x)\right| \leq f(k)$. But $\mathbf{C}_{N}(x)$ is a $\pi$-group, and thus $\mathbf{C}_{N}(x)=\mathbf{C}_{K}(x)$, and the result follows.

\section{Centralizer chains}

Instead of proving Theorem B directly, we prove a somewhat stronger result that lends itself to an inductive argument. To state this theorem, however, we need a somewhat technical definition. Let $N$ act on $H$ via automorphisms and write $\mathcal{C}(N, H)$ to denote the collection of subgroups of $N$ of the form $\mathbf{C}_{N}(X)$ for $N$-invariant subsets $X \subseteq H$. (Observe that that because we require that the subsets $X$ must be $N$-invariant, it follows that each member of $\mathcal{C}(N, X)$ is a normal subgroup of $N$.) We say that a totally ordered subset of $\mathcal{C}(N, H)$ is a centralizer chain, and if $C_{0}>C_{1}>\cdots>C_{r}$ is such a chain, we say that $r$ is its length. Finally, we write $r(N, H)$ to denote the maximum of the lengths of all centralizer chains for the action of $N$ on $H$. Note that if the action of $N$ is nontrivial, then $\mathbf{C}_{N}(1)>\mathbf{C}_{N}(H)$, and this is a centralizer chain of length 1. Thus $r(N, H) \geq 1$ for all nontrivial actions.

Observe that $r(N, H)$ depends only on the permutation action of $N$ on the elements of $H$, and it is independent of the group structure of $H$. It follows that if we use the Hartley-Turull lemma to replace $H$ by an abelian group, this would not change the value of $r(N, H)$.

The promised stronger form of Theorem B is the following.

(3.1) Theorem. Let $N$ be a nilpotent $\pi$-group that acts faithfully on a $\pi^{\prime}$-group $H$. Then there exists $x \in H$ such that

$$
\left|\mathbf{C}_{N}(x)\right| \leq\left(\frac{|N|}{p^{r}}\right)^{1 / p}
$$

where $p$ is the smallest member of $\pi$ and $r=r(N, H)$.

Note that if we apply Theorem 3.1 in the situation of Theorem B, where $N$ is nontrivial, we have $r=r(N, H) \geq 1$, and thus there exists $x \in H$ such that $\left|\mathbf{C}_{N}(x)\right| \leq(|N| / p)^{1 / p}$. In other words, Theorem B really is a consequence of Theorem 3.1.

We begin to work now toward a proof of Theorem 3.1 with an easy lemma that shows how we will use the parameter $r(N, H)$. 
(3.2) Lemma. Let $N$ act on $H$, and write $r=r(N, H)$. Suppose that $\mathcal{X}$ is a collection of $N$-invariant subsets of $H$, and assume that $\mathcal{X}$ is minimal with the property that the action of $N$ on the subset $\bigcup \mathcal{X}$ is faithful. Then $|\mathcal{X}| \leq r$.

Proof. Write $\mathcal{X}=\left\{X_{1}, X_{2}, \ldots, X_{t}\right\}$ and define

$$
C_{i}=\mathbf{C}_{N}\left(\bigcup_{j=1}^{i} X_{i}\right)
$$

for $0 \leq i \leq t$, where we set $C_{0}=N$. The subgroups $C_{i}$ all lie in $\mathcal{C}(N, H)$, and we have

$$
C_{0} \supseteq C_{1} \supseteq C_{2} \cdots \supseteq C_{t} .
$$

If $t>r$, then these containments cannot all be strict, and thus $C_{j-1}=C_{j}$ for some integer $j$ with $1 \leq j \leq t$. In other words, any element of $N$ that centralizes each of the sets $X_{i}$ for $i<j$ also centralizes $X_{j}$. This contradicts the minimality of the collection $\mathcal{X}$, however, because it shows that $X_{j}$ can be deleted, and $N$ will act faithfully on the union of the resulting subcollection. It follows that $t \leq r$, as required.

\section{Proof of the main Result}

In this section we prove Theorem 3.1, and thereby complete the proof of Theorem $\mathrm{B}$. We need the following numerical result.

(4.1) Lemma. Let $x \geq y \geq 1$. Then $y^{x-1} \geq x^{y-1}$.

Proof. It suffices to show that $(x-1) \ln (y) \geq(y-1) \ln (x)$. Now hold $y$ fixed and view both sides of this inequality as functions of $x$ as $x$ varies over the range $y \leq x<\infty$. Since equality holds when $x=y$, it suffices to show that the derivative (with respect to $x$ ) of the left side is at least as large as the derivative of the right side. In other words, it suffices to show that $\ln (y) \geq(y-1) / x$ when $y$ lies in the interval $[1, x]$. Since equality holds when $y=1$, it suffices to hold $x$ fixed and compare the derivatives of both sides with respect to $y$. What we want, therefore, is $1 / y \geq 1 / x$. This is true, of course, since $y \leq x$.

Proof of Theorem 3.1. By the Hartley-Turull lemma, we can assume that $H$ is abelian, and we proceed by double induction, first on $|N|$ and then on $|H|$. If $N$ is trivial, then $r=r(N, H)=0$, and the desired inequality holds. We can assume, therefore, that $N>1$, and thus $r \geq 1$.

Suppose first that $r>1$ and consider a centralizer chain $C_{r}>C_{r-1}>\cdots>C_{0}$ of length $r$. Write $M=C_{1}$, and let $A=\mathbf{C}_{H}(M)$ and $B=[H, M]$, so that $H=A \times B$ by Fitting's lemma. Also, $A$ and $B$ are $N$-invariant since $M \triangleleft N$. Furthermore, if $1 \leq i \leq r$, we know that $M \subseteq C_{i}=\mathbf{C}_{N}\left(X_{i}\right)$ for some subset $X_{i} \subseteq H$, and thus $X_{i} \subseteq A$. Each of the subgroups $C_{i}$, therefore, lies in $\mathcal{C}(N, A)$, and it follows that $r(N / M, A)=r(N, A) \geq r-1$. Also, since $r>1$ by assumption, we have that $C_{0}<M<C_{r}$, and thus $1<M<N$. Finally, since $X_{1} \subseteq A$, it follows that $M$ is the full kernel of the action of $N$ on $A$, and hence $N / M$ acts faithfully on $A$.

Since $M$ centralizes $A$, it must act faithfully on $B$, and thus $r(M, B) \geq 1$ because $M$ is nontrivial. Furthermore, $|M|<|N|$, and so by the inductive hypothesis, there exists an element $b \in B$ such that $v=\left|\mathbf{C}_{M}(b)\right|$ is small. In fact, we can choose $b$ so that $v \leq(|M| / p)^{1 / p}$, where $p$ is the smallest member of $\pi$. 
Also, $N / M$ acts faithfully on $A$ and $|N / M|<|N|$, and so the inductive hypothesis applies to this action too. Since $r(N / M, A) \geq r-1$, we can choose $a \in A$ and set $u=\left|\mathbf{C}_{N / M}(a)\right|$ so that $u \leq\left(|N / M| / p^{r-1}\right)^{1 / p}$. By Lemma 2.1, we have

$$
\left|\mathbf{C}_{N}(a b)\right| \leq u v \leq\left(\frac{|N / M|}{p^{r-1}}\right)^{1 / p}\left(\frac{|M|}{p}\right)^{1 / p}=\left(\frac{|N|}{p^{r}}\right)^{1 / p}
$$

and the proof is complete in this case.

We can now assume that $r=1$, and so $\mathcal{C}(N, H)=\{1, N\}$, and our goal in this case is to find $x \in H$ such that $\left|\mathbf{C}_{N}(x)\right| \leq(|N| / p)^{1 / p}$. Let $H / A$ be an $N$ composition factor of $H$, and note that $\mathbf{C}_{N}(A) \in \mathcal{C}(N, H)$. If $\mathbf{C}_{N}(A)=1$, then $N$ acts faithfully on $A$, and since $A<H$, the result follows by the inductive hypothesis on $|H|$. The only other possibility is that $\mathbf{C}_{N}(A)=N$, and hence $A=\mathbf{C}_{H}(N)$ and we can write $H=A \times B$, where $B=[H, N]$, and $N$ acts faithfully on $B$. Again, we are done by the inductive hypothesis if $B<H$, and so we can assume that $B=H$ and $A=1$. In other words, we are reduced to the case where $H$ is a simple $N$-module in characteristic not dividing $|N|$.

Suppose now that every abelian normal subgroup of $N$ is cyclic. Since $N$ is nilpotent, every Sylow subgroup of $N$ satisfies the same hypothesis, and it follows that the odd Sylow subgroups are cyclic and that the Sylow 2-subgroup of $N$ is either cyclic, or else is dihedral, semidihedral or generalized quaternion. In any case, we see that $N$ has a cyclic subgroup $C$ of index not exceeding 2 .

If $1 \neq x \in H$, then $\mathbf{C}_{C}(x)$ is characteristic in $C$, and hence is normal in $N$. It follows that $\mathbf{C}_{H}\left(\mathbf{C}_{C}(x)\right)$ is a nonidentity $N$-invariant subgroup of $H$, which must, therefore, be all of $H$. Thus $\mathbf{C}_{C}(x)$ acts trivially on $H$, and we conclude that $\mathbf{C}_{C}(x)=1$, and hence $C \cap \mathbf{C}_{N}(x)=1$. Therefore $\left|\mathbf{C}_{N}(x)\right| \leq|N: C| \leq 2$. If $C=N$, then $\left|\mathbf{C}_{N}(x)\right|=1 \leq(|N| / p)^{1 / p}$, as required. Otherwise, $|N|$ is even, and hence $p=2$. In this case, $|N| \geq 8$ and we have $\left|\mathbf{C}_{N}(x)\right| \leq 2 \leq(|N| / 2)^{1 / 2}$, and we are done in this case also.

Finally, we can suppose that $N$ has a noncyclic abelian normal subgroup, and hence it is imprimitive in its action on the simple module $H$. It is therefore possible to write $H$ as a nontrivial direct sum of a collection $\mathcal{X}$ of subgroups transitively permuted by the action of $N$. Choosing $\mathcal{X}$ so that $|\mathcal{X}|=q$ is as small as possible, we note that $q$ is the index in $N$ of the stabilizer $R$ of one of the members of $\mathcal{X}$. Also, $R$ is a maximal subgroup of $N$, and hence $R \triangleleft N$, and thus $R$ stabilizes all of the subgroups in $\mathcal{X}$. Every subgroup of $N$ not contained in $R$ therefore acts transitively on $\mathcal{X}$. Also, $R>1$ since $N$ is noncyclic.

Suppose first that $r(R, H)<q$. By Lemma 3.2, it follows that $R$ acts faithfully on the sum $K$ of $q-1$ of the members of $\mathcal{X}$. Since $K<H$ and $r(R, K) \geq 1$ because $R>1$, the inductive hypothesis applies, and we can find $x \in K$ such that $\left|\mathbf{C}_{R}(x)\right| \leq(|R| / p)^{1 / p}$. Because $x \in K$, the component of $x$ in at least one of the direct summands in $\mathcal{X}$ is trivial, and yet $x$ is nontrivial. It follows that $\mathbf{C}_{N}(x)$ cannot permute the set $\mathcal{X}$ transitively. Thus $\mathbf{C}_{N}(x) \subseteq R$ and $\left|\mathbf{C}_{N}(x)\right|=\left|\mathbf{C}_{R}(x)\right| \leq$ $(|R| / p)^{1 / p} \leq(|N| / p)^{1 / p}$, as required.

We can now assume that $r(R, H) \geq q$, and thus by the inductive hypothesis (on $|N|$ ), we can find $x \in H$ such that $\left|\mathbf{C}_{R}(x)\right| \leq\left(|R| / p^{q}\right)^{1 / p}$. Since $\left|\mathbf{C}_{N}(x)\right| \leq$ $|N: R|\left|\mathbf{C}_{R}(x)\right|$ and $|N: R|=q$, we have

$$
\left|\mathbf{C}_{N}(x)\right|^{p} \leq q^{p}\left|\mathbf{C}_{R}(x)\right|^{p} \leq q^{p}\left(\frac{|R|}{p^{q}}\right)=\frac{q^{p}|N|}{q p^{q}}=\left(\frac{|N|}{p}\right)\left(\frac{q^{p-1}}{p^{q-1}}\right) \leq \frac{|N|}{p},
$$


where the final inequality follows from Lemma 4.1 since $p \leq q$. The result now follows.

\section{FURTHER REMARKS}

We have no evidence that the hypothesis that $N$ is nilpotent is really necessary in Theorem B. It seems that if $N$ acts coprimely and faithfully on $H$, it should at least be true that there exists an element $x \in H$ such that $\left|\mathbf{C}_{N}(x)\right| \leq|N|^{1 / 2}$, but we are unable to to prove this even if $N$ is assumed to be solvable.

In the case where the acting group $N$ is abelian, we can take $\pi$ to be empty in Theorem $\mathrm{C}$, and we deduce that there exists $x \in H$ such that $\mathbf{C}_{N}(x)=1$. It is easy to prove this directly by using the Hartley-Turull lemma to replace $H$ by an abelian group, but there is another elementary proof available that is perhaps not as well known as it deserves to be. It is possible to obtain the result by using a variation on Brodkey's theorem [1], applied to the semidirect product $G=H N$.

Every conjugate of $N$ in $G$ has the form $N^{x}$ with $x \in H$, and thus it suffices to find such a conjugate that intersects $N$ trivially. Note that Brodkey's theorem applies since $G$ is $\pi$-separable, and so every subgroup satisfies property $D_{\pi}$. Also, $\mathbf{O}_{\pi}(G)=1$ because $N$ acts faithfully on $H$.

(5.1) Theorem (Brodkey). Let $N$ be an abelian Hall $\pi$-subgroup of a group $G$ and assume that every subgroup of $G$ satisfies property $D_{\pi}$. If $\mathbf{O}_{\pi}(G)=1$, then $N \cap M=1$ for some $G$-conjugate $M$ of $N$.

Proof. Choose a conjugate $M$ of $N$ such that $D=M \cap N$ is minimal, and assume that $D>1$. Since $\operatorname{core}_{G}(N)=1$, we can choose some conjugate $Q$ of $N$ such that $D \nsubseteq Q$. Now let $U=\langle N, M\rangle$ and note that $D \triangleleft U$ since $M$ and $N$ are abelian. Also, for some element $u \in U$, we have $(U \cap Q)^{u} \subseteq M$ by the $D_{\pi}$ property in the subgroup $U$. Then $N \cap Q^{u}=N \cap U \cap Q^{u} \subseteq N \cap M=D$. By the minimality of $D$, we deduce that $N \cap Q^{u}=D$, and thus $D^{u}=D \subseteq Q^{u}$. This is a contradiction, however, because $D \nsubseteq Q$.

\section{REFERENCES}

1. J. S. Brodkey, A note on finite groups with an abelian Sylow group, Proc. Amer. Math. Soc. 14 (1963), 132-133. MR 26:200

2. B. Hartley and A. Turull, On characters of coprime operator groups and the Glauberman character correspondence, J. Reine Angew. Math. 451 (1994), 175-219. MR 95d:20010

3. D. S. Passman, Groups with normal solvable Hall p'-subgroups, Trans. Amer. Math. Soc. 123 (1966), 99-111. MR 33:4143

Department of Mathematics, University of Wisconsin, 480 Lincoln Drive, Madison, WISCONSIN 53706

E-mail address: isaacs@math.wisc.edu 